\title{
Mechanistic Mathematical Modelling of Pothole Development from Loss of Roadway Subsurface-Materials
}

\author{
Okechukwu Joseph Ifeanyi Ezekwesili*, Jonah Chukwuemeka Agunwamba \\ Department of Civil Engineering, Faculty of Engineering, University of Nigeria, Nsukka 410001, Enugu State, Nigeria
}

Corresponding Author Email: okecylo@gmail.com

https://doi.org/10.18280/mmep.080202

Received: 31 August 2020

Accepted: 5 January 2021

\section{Keywords:}

mechanistic mathematical model, pothole, internal-erosion, loss of roadway subsurface materials, traffic load pressure, excess porewater pressure, pumping out of particles

\begin{abstract}
In this paper, we developed a mechanistic mathematical model. It implies the engineering problem of pothole development on roadways. The issue involves internal erosion, a decline of subsurface materials, voids creation, depression, materials damage, and potholes' appearance on the road's surface. Our study aims to predict why, how, and when pothole develops from the loss of roadway subsurface materials. We reviewed many sources as our first method. It involved using and adapting the guiding principles for migrating particles upwards. We then changed specific parameters, formulated our model equation, solved it using the separation of variables, and then verified it. Observations from our review show that high traffic load pressure and water must be present on the road for particle migration to occur. They generate excess pore-water pressure that enables the movement of particles upwards. Particle relocation causes voids and dislocation of materials. Results show that an increase in time, cracks, soil erosion coefficient, and a decrease in the roadway's height led to a rise in the number of materials lost from the pavement. Our study is relevant because it will better inform road managers and modelers on potholes, and they can-do preventive measures to avert total road failure.
\end{abstract}

\section{INTRODUCTION}

Roadways are infrastructures used to convey goods with both living and nonliving creatures. Studies show that pothole occurrences on these roadways are a known engineering problem [1-4]. It reduces the lifespan of these roadways. Studies have shown that a headway for facing these potholes is to predict them $[1,2]$. According to research, by using differential equations, we can simulate real-life engineering issues and predict how they perform [5-10].

Using mechanistic mathematical models may then predict the development of these potholes on roads. It requires applying various specific data of the roadway's subsurface and surface materials, and then different established principles. Research has shown that using detailed data, and established principles draw the prediction that makes a road deformation model closer to reality $[11,12]$.

Applying mechanistic mathematical models to predict potholes might have been a puzzle to the road, civil, geotechnical, water, and other engineers. It might have been a puzzle because, unlike empirical models, mechanistic models use established principles in differential equations to model how such events happen. The issues modeled in this paper involves the loss of roadway subsurface materials by their migration upwards to the roadway's topmost layers. Researches show that it results in voids formation in the sublayers of the roadway's surface and further develops into a roadway deformation called a pothole $[1,2,12]$.

This study is essential to engineers and modelers because by predicting pothole occurrence with the aid of derived differential equations, the following should happen. Roadways may not fail early because of prompt preventive measures. Second, understanding of the pothole phenomenon is better. Last, the usage of relevant data to represent the mathematical essence of the pothole occurrence is better.

Investigations have shown that potholes, warping, rutting, raveling, edge-break, cracks, and shear failures are common types of road deformation [1, 2, 4, 13]. During road deformation (pothole) modeling, most research focused on the phenomena that affect the top layers of the road [7, 2, 14-17]. It is because modeling the road's subsurface activities is tricky since it requires roadway subsurface data and different principles. Studies assumed the roadway surface (asphalt layers) to be plates, beams, or slabs resting on foundations [2, 7, 14-17]. Using those assumptions may not describe what happens below the surface layers. Research show that for potholes to occur on a roadway, water pressure and traffic-load should be present $[2,12,13,18-20]$. Their presence leads to a rise in pore-water pressure. It then leads to internal erosion and the upwards migration of subsurface materials.

Studies have shown that modeling the movement of particles upwards consider several principles $[1,2,7,12,18$, 20-27]. The principles examined were mud-pumping, advection, internal-erosion, solute transport, pore-water pressure, suction, suffusion, pore fluid viscosity, and roadwaymaterials breakdowns under both traffic-loads and water movement.

Several researchers have studied the phenomenon that involves the pumping out, migration, or movement of particles from the road's subsurface upwards $[1,4,18,19,20,22,23$, 25-28]. Most of these researches did not relate their findings directly to pothole development's engineering problem on 
roads. Some of them agree that underlying materials move upwards through the existing cracks, pores, and joints of different structures to their layers' surface. They become mud or particles accumulating on those surfaces. These moving particles could be related to an eroded roadway's subsurface materials, subgrade soil, or foundation particles below the asphalt surface layers. A typical roadway comprises asphalt layers ontop, stone base layer just below, and then soil layers further below.

Several pieces of literature know internal-erosion as subsurface-erosion or piping-erosion [19, 20, 26, 29-35]. It involves the internal scouring, loss, and redistribution of soil or subsurface materials underneath structures like roads, dams, and others because of fluid (water) and load pressure met by those structures.

From studies many factors influence the pumping out or upwards migration and internal-erosion occurrences $[1,4,18$ $20,22,23,25-36]$. These factors are the fluid (water) presence and velocity, applied traffic-load, the porosity of the roadway's subsurface materials, pore-water pressure, pore fluid viscosity, pore sizes, particle size distribution, pavement's height, and soil erodibility coefficient. Also, a study from [20] suggests a reduction of the number of particles deposited may occur over time because of a decrease in the stone base layer permeability.

Many types of research show that predicting and tackling potholes is necessary $[1,2,20]$. They stated that we need to do proper roadway design, maintenance, construction, suitable asphaltic concrete mix design, geotextiles, drainage, and other relevant construction materials and techniques in addressing potholes after the forecast. They stated that poor implementation of the above is the reason for pothole development.

Pothole deformation occurs as bowl-shaped holes of many sizes in the roadway surface. Studies described why and how potholes occur [1-4]. They stated that potholes occur because of the application of excess traffic-load and water present on the road. They also stated that subsurface and surface materials deform by displacement, depression, and breakage into small pieces by constant traffic-load pressure, cracking, fatigue, migration upwards, loss, or disintegration of their mixtures. They stated the following as the procedure for potholes in development. First, cracks form on the roadway. Second, water infiltrates the roadway-subsurface through those cracks and groundwater upward movement. The roadway subsurface becomes saturated. Applying more traffic load increases the excess pore-water pressure. It then results in internal-erosion and migration of the roadway's subsurface materials upwards through those cracks and pores. Under more pressure, these materials go to the road's surface and its layers' topmost parts. It leaves behind voids underneath the pavement's surface. After, the pothole appears on the roadway surface under extra pressure.

Also, many researches and works have established that weak (subsurface, surface, subgrade and/or underlying) materials affect the roadway's lifespan $[1-4,7,12,14,20,26$, 37]. They also showed that under excess traffic-load pressure and in the presence of water, the pore-water pressure increases. The number of particles pumped out depends on the properties of the subsurface roadway-materials. For example, too many fines in the roadway's subsurface promote internal-erosion. Internal-erosion produces and allows pumping out of materials. Pumping out of materials promotes voids formation and then potholes.
This paper aims to predict and describe, using a mechanistic mathematical model, why, how, and when potholes occur on a roadway by indicating the number of particles lost from under it. It intends to relate the number of particles lost to the pothole developed. This model expects to compute the number of roadway subsurface materials lost or pumped out upwards when we have a change in excess pore water pressure, time, soil erosion coefficient, and the roadway's height. Another intention is to account for the number of displaced, lost, and deposited materials in the roadway's stone-base and its surface.

\section{METHODOLOGY}

We used the approach stated here to achieve our aims and objectives. First, we examined various literature to find applicable principles and equations for us. Our review identified several principles and equations.

Next, we then used the equations governing the upwards movement of particles from the study [20] since it better suits our study's aim. We then adopted the one-dimensional advection-dispersion-solute-transport equation used by Kermani [20] because it is closer to our purpose.

Later, we considered various assumptions, conditions, variables, and data to ensure that the adopted equation best fits our model's purpose.

More, we decoupled the adopted equation. It produced two equations, the particle mobilization, and deposition equations. After, we selected and modified the particle deposition equation to represent our pothole model because it contains our model's purpose.

Later, we solved our model equation using the separation of variables method. We used adapted data gotten from experience, a Scaled Accelerated Road Laboratory Test conducted by Kermani [20], and other literature to verify and test our model's abilities. In verifying our model, we studied the effects of a change in excess pore water pressure, time, coefficient of soil erosion, and the road's height. Last, we presented our results and conclusions.

\section{FORMULATION OF THE MODEL EQUATION}

\subsection{Conceptualizing and visualizing of model equation}

Imagine that a road has developed cracks under the application of excess daily traffic. These cracks deepened and widened over time. The roadway becomes a porous medium because fluids can move in and out of it. Water filled the roadway's subsurface layers through those cracks and groundwater movement. The soil layers in the road foundation soften and brakes into particles. These particles mix up with the water to become mud and fluid. The road foundation becomes deformed or out of place. It shows that the initial mobilization of particles has occurred. An initial internalerosion and pore-water pressure trigger these particles. More traffic-loading exerts on the roadway and applies pressure. The added load and significant stress increase the pore-water pressure, and it becomes excess. Upwards migration of the mobilized particles commences because of the extra porewater pressure. The whole process beginning from the infiltration of water to the upwards movement of particles recycles.

The particles further migrate to the top part of the stone base 
layers passing through the existing cracks and pores. The size and rate of upwards migration keep increasing until it pumps out of the pavement through those cracks. The particle deposition on the surface and top part of the stone base layers continues. The pores did not clog up on time because some materials pumped out to the road's surface. The particles' small size and fluidity made a move freely and upwards through the pavement's cracks and pores quickly. The particles lost from beneath the road's surface to the surface and top part of the stone base layers create vacuums. They did not reenter the subsurface of the pavement. Water washed these materials off. Also, some of these materials remain in the spaces existing in the asphaltic and stone base layers. Voids keep developing, reoccurring, and widening beneath the road's surface. After many hours, days, weeks, months, or years, the created holes underneath the road's surface become threats. Any further load deforms the asphaltic layer by breaking and depressing it. The damaging of the asphaltic layer materials continues and recycles until it takes the form of the voids created under it and becomes a visible pothole.

In summary, in the presence of infiltrated water into a road's subsurface through existing cracks and pores, and under traffic-load pressure, internal-erosion and pore-water pressure occur, the road loses many subsurface particles that leave behind voids, and the road surface depresses into those vacuums until they become visible as potholes on the road's surface. The number of materials lost is directly proportional to the potholes because of their loss that translates to voids and later potholes. Our concept strives to predict the number of materials lost, and from past works, the principle of the one- dimensional advection-dispersion-solute-transport equation governing the migration of subgrade particles into subbase adopted from the study [20] best fits our model aim. It accounts for internal erosion, flows through a porous medium, pumping out, and migration of soil fines upwards into the road's subbase or stone-base Eq. (1) shows the adapted equation.

Our model took steps further by predicting and accounting for the total number of materials lost by their deposition in the top part of the stone base and on the pavement's surface. It related it to pothole development.

$$
\frac{d C p}{d T}+D \frac{d^{2} C p}{d X^{2}}+v \frac{d C p}{d X}+\lambda V C p-\beta\left(V-V_{c r}\right)=0
$$

The used parameters and variables in Eq. (1) are as follows. The $\mathrm{Cp}$ is the particle mass concentration in pore fluid. $\mathrm{T}$ is time. $\mathrm{X}$ is distance. $\mathrm{D}$ is the longitudinal dispersion coefficient. The $\lambda$ is the filtration coefficient, and it accounts for the particle deposition process. $\mathrm{V}$ is the water seepage velocity. The $\beta$ is the soil erosion coefficient, and it denotes particle detachment. The Vcr is the critical water seepage velocity.

We presented Figures 1, 2, 3, and 4 below. These figures show visual views of water infiltration processes and the effects of cyclic and no cyclic traffic loading on the roadway's subsurface layers. More, they show internal erosion, mobilization, pumping out upwards, and deposition of particles in the stone base and on the surface of the roadway. All further show the loss of the roadways subsurface materials that promote the void formation and pothole occurrence.
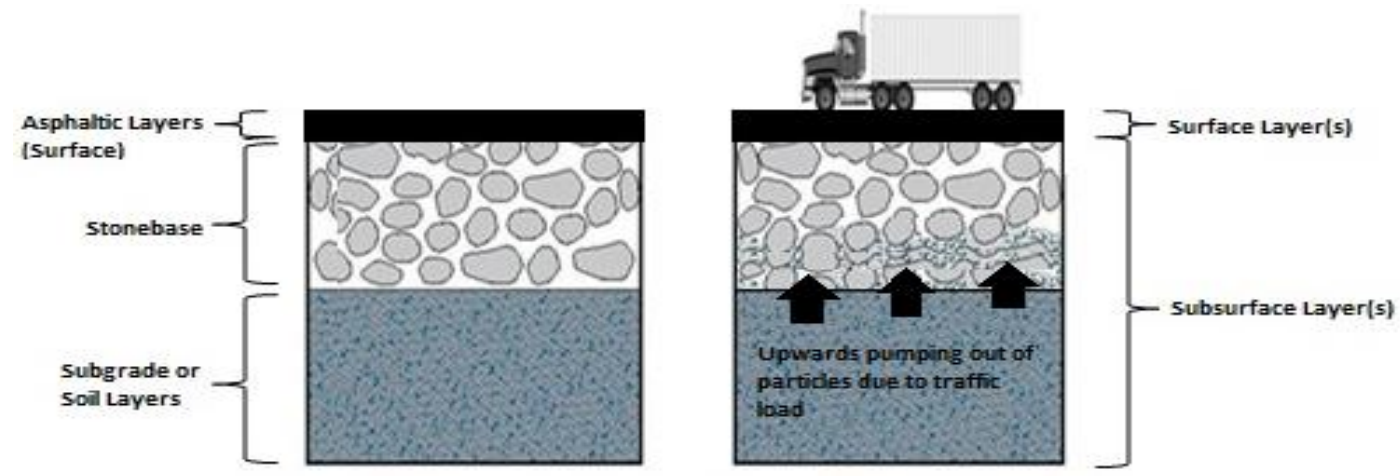

Figure 1. Effects of no traffic loading on the lefthand side, while the pumping out upwards of eroded particles from the road's subsurface layers because of traffic load is on the righthand side

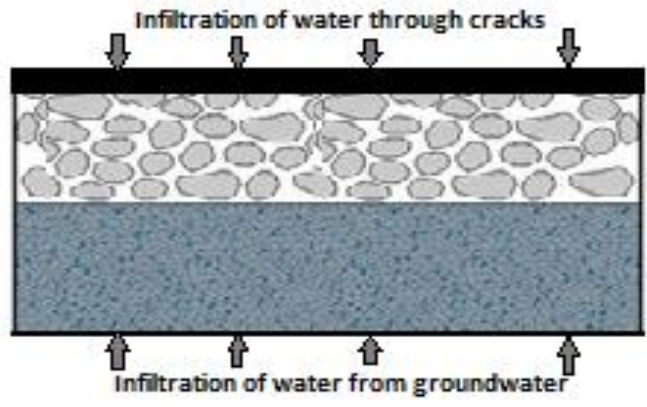

(a)

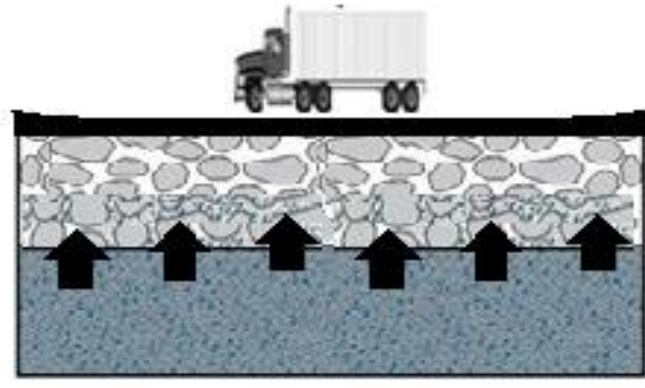

(b)

Figure 2. Infiltration of water into road subsurface is on the lefthand side, while the generation of pore water pressure, internalerosion, and initial pumping out of eroded particles upwards into upper layers because of the exerted load are on the righthand 

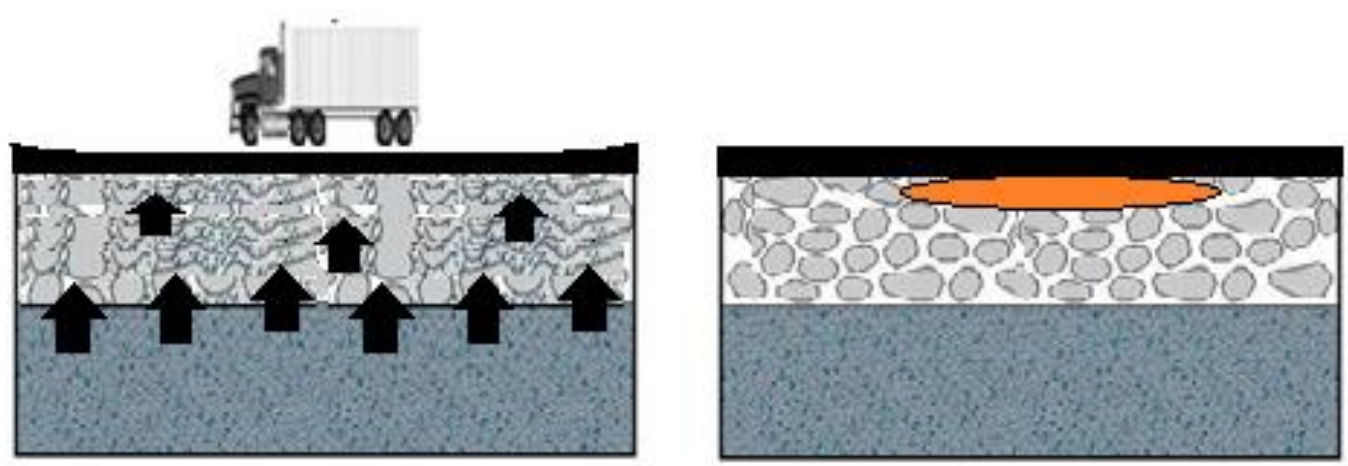

Figure 3. Further pumping out of particles upwards to the asphalt's surface and stone-base layers is on the lefthand side, while voids growth (oval shape) below the asphalt layer is on the righthand side

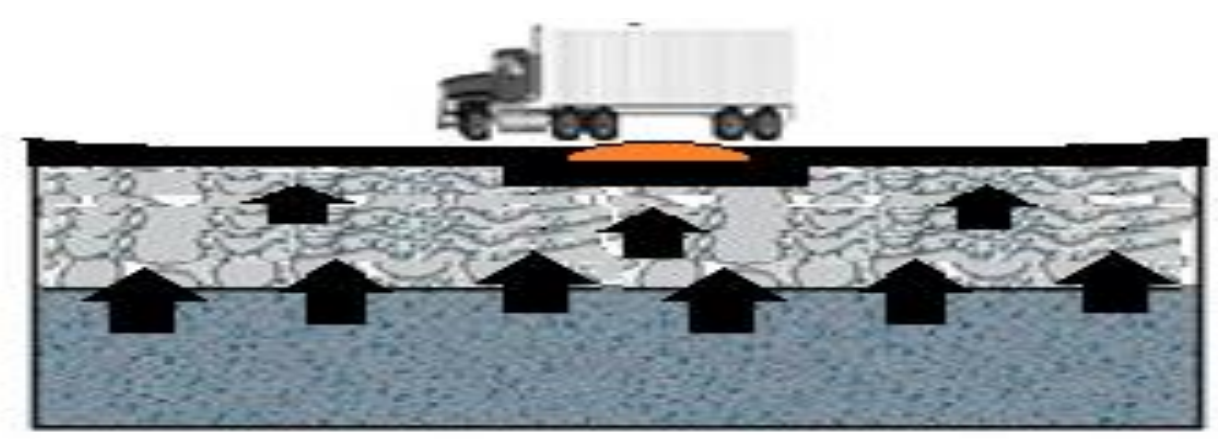

Figure 4. Depression of asphalt top layer into underneath voids and presence of the pothole on the road surface (oval shape) under exertion of more load

\subsection{Assumptions}

We made certain assumptions to enable us to modify Eq. (1), formulate our pothole model equation, and suit our model's aim. We listed these assumptions below to assist us.

(1) Deep cracks exist in the roadway because of the past and repeated excess applied traffic-load.

(2) Fluid (water) entered the roadway's subsurface materials from cracks and groundwater movement.

(3) We assumed the roadway (Wearing + Binder + Stonebase + top part of the soil layers) structure as one homogenous layer and a porous medium because of cracks and pores.

(4) The number of particles lost equals the volume of voids created and then equal to the pothole volume.

(5) The system is non-dispersive, $\mathrm{D}=0$.

(6) There is an initial particle concentration in the pore fluid after particle-mobilization caused by internalerosion and before upwards particles-transport and deposition.

(7) Clogging of pores did not occur rapidly during continuous pumping of fines because the existing deep cracks in the roadway allowed the materials-deposition on the roadway surface.

(8) Pumping out of materials may only occur for 365 days in a cycle, so we assume any other occurrence occurs in a new year cycle.

(9) The materials deposited on the roadway's surface, transported and washed off the area and did not reenter the roadway's sub-layers.

\subsection{Model conditions}

We gave the following conditions for this model.
At $\mathrm{T}=0, \mathrm{C}=0$

At $\mathrm{X}=0, \mathrm{~T}>0, \mathrm{C}=\mathrm{C} 0$.

At $\mathrm{X}>0,0<\mathrm{T} \leq 365$ days, Constant $\mathrm{B}=\lambda^{*} \mu$, and constant $\mathrm{K}=-\lambda / \mathrm{V}$.

\subsection{Parameterization of pothole model equation}

Considering that this paper's target is to develop a mechanistic mathematical pothole development model, we used specific parameters.

We used the following parameters listed below.

(1) We replaced $C p=$ the particle mass concentration in pore fluid in Eq. (1) with $C=$ the number of materials lost in Eq. (3a).

(2) We adapted a value for $\mu=$ dynamic viscosity of porefluid from the study [20], but we can measure it using a DV3TLV rheometer.

(3) We got some of the presumed values for $\Delta P=$ excess dynamic pore water pressure from the study [20], but we can get it using readings of dynamic pore water pressure cells embedded in the roadway's subsurface layers during the scaled pavements laboratory test.

(4) We used $T$ to show the time.

(5) The adopted formula for $E t=$ erosion rate of the subgrade soil is from the study [20]. Eq. (2a) shows the formula.

$$
E t=\beta(\tau t-\tau c r)
$$

(6) We assumed some values for $\beta=$ coefficient of soil erosion from [20], but we can get it by doing a holeerosion test on soil samples.

(7) The presumed $V=$ water seepage velocity is from the study [20]. 
(8) We presumed $V c r=$ shows critical water seepage velocity from the study [20].

(9) The assumed $\tau c r=$ critical hydraulic shear stress beyond which we have particle mobilization is from the study [20]. We can get it by doing a hole-erosion test on soil samples.

(10) We presumed $\tau=$ hydraulic shear stress and the formula from the study [20]. Eq. (2b) shows the formula.

$$
\tau=\frac{\Delta P r}{2 X}
$$

(11) The adapted $\lambda=$ filtration coefficient; it relates to particle-deposition. We adapted it and the formula from the study [38]. Eq. (2c) shows the formula.

$$
\lambda=N \frac{R^{2}}{8 \mu X}
$$

(12) We assumed $N=$ number of deep pores and cracks from practice on site.

(13) The adapted value for $R=$ radius of the flow tube is from the study [20], but we can get it by measuring the most massive, eroded particles.

(14) We assumed $X=$ roadway's height between the asphalt surface and soil layers or the road's thickness. It accounts for distance traveled by particles upwards.

(15) The $C_{0}=$ first materials lost. We got it and the formula from the study [20]. Eq. (2d) shows the formula.

$$
C_{0}=\frac{M}{Q * T}
$$

(16) We adapted $M=$ mass of the eroded soil and its formula from the study [20]. Eq. (2e) shows the formula.

$$
M=\left(E_{t} * A * T\right)+M_{0}
$$

(17) The $Q=$ Flow rate of the eroded soil. We adapted it and the formula from the study [20], as shown in Eq. (2f).

$$
Q=\pi \frac{\Delta P R^{4}}{8 \mu X}
$$

(18) We adapted $C_{2}=$ the number of materials lost but trapped in the top part of the stone base and not deposited on the pavement's surface, and its formula is from the study [20]. Eq. (2g) shows the formula.

$$
C_{2}=\left\{C_{o} \exp ^{-\frac{\lambda}{V} X} * \lambda\right\} *\left(t-\frac{X}{V}\right)
$$

(19) We adapted $A=$ tire contact area from [20].

(20) We used $C_{(X, T)}=$ the total number of materials lost. These were materials deposited on the roadway's surface and trapped in the stone base's top part.

\subsection{Derivation of pothole model equation and solution}

The modification started from Eq. (3a), as seen below. First, we applied the assumption of the system being non-dispersive that is $D=0$ to Eq. (3). This assumption modifies the advection-dispersion-solute-transport equation into Eq. (3a) below. Later, we decoupled Eq. (3a). It represented particle mobilization and particles-deposition as adapted from the study [20]. Eqns. (3b), and (3c) shows the decoupled equations as particle mobilization and deposition, respectively.

$$
\begin{gathered}
\frac{d C p}{d T}+D \frac{d^{2} C p}{d X^{2}}+v \frac{d C p}{d X}+\lambda V C p-\beta\left(V-V_{c r}\right)=0 \\
\frac{d C}{d T}+V \frac{d C}{d X}+\lambda V C-\beta(v-v c r)=0 \\
\frac{d C}{d T}+V \frac{d C}{d X}-\beta(v-v c r)=0 \\
\frac{d C}{d T}+V \frac{d C}{d X}+\lambda V C=0
\end{gathered}
$$

Considering Eq. (3c) above, we then modified it as our pothole development model to predict the number of roadway subsurface materials deposited on the roadway's top surface and stone base layers through the existing cracks and pores acting as the porous media. After, we equated the materials lost to the void created underneath the roadway asphaltic layers. These voids transfer over time to the roadway's surface, resulting in potholes. We did not consider Eq. (3b) because our assumptions say that the materials' mobilization has happened. The derived mechanistic mathematical pothole development model after modification of Eq. (3c) thus becomes as seen in Eq. (4) below.

$$
\frac{1}{V} \frac{d C}{d T}+\frac{d C}{d X}+\lambda C=0
$$

To solve Eq. (4) above, we simplified further by differentiating it to $T$. Eq. (5a) shows the further simplified equation. Eqns. (5b), (6a), (6b), and (6c) show the Application of the separation of variables technique.

$$
\frac{1}{V} \frac{d^{2} C}{d T^{2}}+\frac{d C}{d X}+\lambda \frac{d C}{d T}=0
$$

Let us separate the variables to get Eq. (5b). We then apply Eq. (5b) in Eq. (5a) to have Eq. (6a).

$$
\begin{gathered}
C=X_{X} * T_{T} \\
\frac{1}{V} \frac{T^{I I}}{T}+\frac{X^{I}}{X}+\lambda \frac{T^{I}}{T}=0
\end{gathered}
$$

Let's equate Eq. (6a) above to $-\mathrm{K}$ and then equate the two variables to solve it efficiently. Eqns. (6b), and (6c) shows the effect of equating Eq. (6a) to $-\mathrm{K}$.

$$
\begin{gathered}
\frac{1}{V} \frac{T^{I I}}{T}+\lambda \frac{T^{I}}{T}=-K \\
-\frac{X^{I}}{X}=-K
\end{gathered}
$$

Let us solve for $T_{(T)}$ by equating Eq. (6b) to 0. Eq. (7) show the effects of equating it to 0 .

$$
\frac{1}{V} \frac{T^{I I}}{T}+\lambda \frac{T^{I}}{T}+K=0
$$


Let us multiply Eq. (7) above by $V T$ and simplify it further. Eq. (8) shows the effects of doing the above.

$$
T^{I I}+\lambda V T^{I}+K V T=0
$$

From the auxiliary equation, as seen in Eq. (9a), let $\mathrm{a}=1, \mathrm{~b}$ $a=1 ; b=\lambda V ; c=K V$. Eq. (9b) shows the ideal solution that suits Eq. (9a) while we see its typical roots in Eq. (9c).

$$
\begin{gathered}
a m^{2}+b m+c=0 \\
T_{T}=A \cos m T+B \sin m T \\
m=\frac{-\lambda V \pm \sqrt{(\lambda V)^{2}-(4 * 1 * K V)}}{2 * 1}
\end{gathered}
$$

So, simplifying Eq. (9c) further, we have Eqns. (10a) and (10b).

$$
\begin{aligned}
& m=\frac{-\lambda V-\sqrt{(\lambda V)^{2}-(4 K V)}}{2} \\
& m=\frac{-\lambda V+\sqrt{(\lambda V)^{2}-(4 K V)}}{2}
\end{aligned}
$$

Substituting Eqns. (10a) and (10b) in Eq. (9b) thus gives.

$$
\begin{aligned}
& T_{(T)} \\
& =A \cos \left[\frac{-\lambda V-\sqrt{(\lambda V)^{2}-(4 K V)}}{2}\right] * T \\
& +B \sin \left[\frac{-\lambda V+\sqrt{(\lambda V)^{2}-(4 K V)}}{2}\right] * T
\end{aligned}
$$

Applying the first condition of $C_{(x, 0)}=0$ in Eq. (11) above, we thus have.

$$
0=A * 1+B * 0
$$

If $A=0$ from Eq. (12) after solving, this means that $B \neq$ 0 so as not to have a trivial solution.

So, we thus have Eq. (13) after plugging values of $A=0$ and $B \neq 0$ into Eq. (11).

$$
T_{(T)}=B \sin \left[\frac{-\lambda V+\sqrt{(\lambda V)^{2}-(4 K V)}}{2}\right] * T
$$

Let us multiply both sides of Eq. (6c) by $-X$. We obtained Eq. (14) after the multiplication.

$$
X=K X
$$

Integrating and solving Eq. (14) above, we thus have.

$$
\begin{gathered}
\log X=e^{K X}+\log F_{1} \\
X_{X}=F_{1} \exp ^{K X}
\end{gathered}
$$

Applying the second condition of $C_{(0, T)}=C_{(0)}$ in Eq. (15b) we thus have Eqns. (16a) and (16b) afterward.

$$
C_{o}=F_{1} * \exp ^{K(0)}
$$

$$
F_{1}=C_{o}
$$

Plugging back the values of Eq. (16b) into Eq. (15b) we thus have Eq. (17).

$$
X_{X}=C_{o} \exp ^{K X}
$$

We put in values of $T$ from Eq. (13) and $X$ from Eq. (17) into Eq. (5b) to get Eq. (18).

$$
C_{(X, T)}=C_{o} \exp ^{K X} * B \sin \left[\frac{-\lambda V+\sqrt{(\lambda V)^{2}-(4 K V)}}{2}\right] T
$$

Let us use the third condition. Recall that we differentiated Eq. (4) with $T$. Now, using the third condition of when $X>0$, $0<\mathrm{T} \leq 365$ days, Constants $\mathrm{B}=\lambda^{*} \mu$, and Constant $\mathrm{K}=-\lambda / V$, so we integrate Eq. (18) with $T$. Let $C_{(X, T)}=C_{3}$. Also, let $C_{2}$ account for the number of materials lost but trapped in the top part of the stone base and not deposited on the pavement's surface. The Eq. (19) shows our obtained solution.

$$
\begin{gathered}
C_{3}=\left(\left\{C_{o} \exp ^{-\frac{\lambda}{V} X} * \lambda \mu *-\cos \left[\frac{-\lambda V+\sqrt{(\lambda V)^{2}+(4 \lambda)}}{2}\right.\right.\right. \\
* T]\})+C_{2}
\end{gathered}
$$

Eq. (4) above shows the mechanistic mathematical model equation that we derived. However, Eq. (19) above shows the derived solution arrived when we use the method of separation of variables.

\section{RESULTS AND DISCUSSIONS}

We verified our developed model using specific data got and adapted from several works of literature, experience, and an already conducted Scaled Accelerated Roadway Laboratory Test, measurements, and geotechnical investigation. The data were both varied and kept constant at various times. Some values did not change during the verification, and they were $A=0.00386 \mathrm{~m}^{2}, \mathrm{~V}=1 \mathrm{~mm} . \mathrm{s}^{-1}, \mathrm{R}$ $=0.003 \mathrm{~m}, \mathrm{~N}=10$, and $\mu=0.0029 \mathrm{~N}$. $\mathrm{s} . \mathrm{m}^{-2}$. A negative or zero value for the number of materials lost indicates that no potholes developed, while a positive value describes that potholes developed on the roadway. Figures 5, 6, 7, and 8 show the effects of a change in values for excess pore water pressure, time, coefficient of soil erosion, and the height of the roadway on the number of materials eroded and later lost or pumped out.

\subsection{Influence of excess dynamic pore pressure on the model's performance}

We inputted different values for excess dynamic pore water pressure in the model while keeping other parameters at the same value. We kept the roadway's height at $0.3 \mathrm{~m}$, the soil erosion coefficient at $0.00058 \mathrm{s.m} \mathrm{m}^{-1}$, and the time interval at 180 days. Figure 5 shows the sensibility of the model to the different input values for excess pore water pressure. The result indicates an increase in the total number of materials lost from the roadway's subsurface as the values for excess pore water pressure increase. 


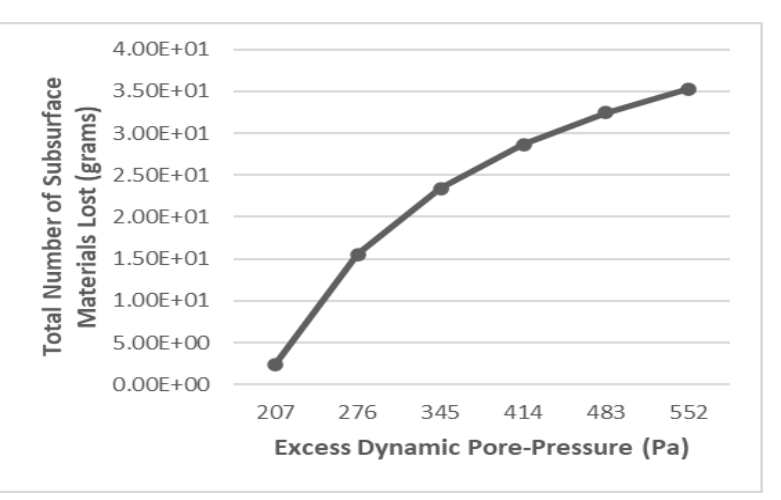

Figure 5. Effect of excess pore pressure on the total number of subsurface materials lost

\subsection{Influence of time on the model's performance}

We increasingly varied time alongside the excess pore water pressure values while keeping other parameters at the same value. The inputted value for the height of the roadway was $0.3 \mathrm{~m}$. The entered value for the coefficient of soil erosion was $0.00058 \mathrm{~s} . \mathrm{m}^{-1}$. Figure 6 shows the effect of varying time alongside pore water pressure on the total number of materials lost from the roadway's subsurface. The result shows that an increase in time causes a rise in the total number of materials lost from the roadway's subsurface.

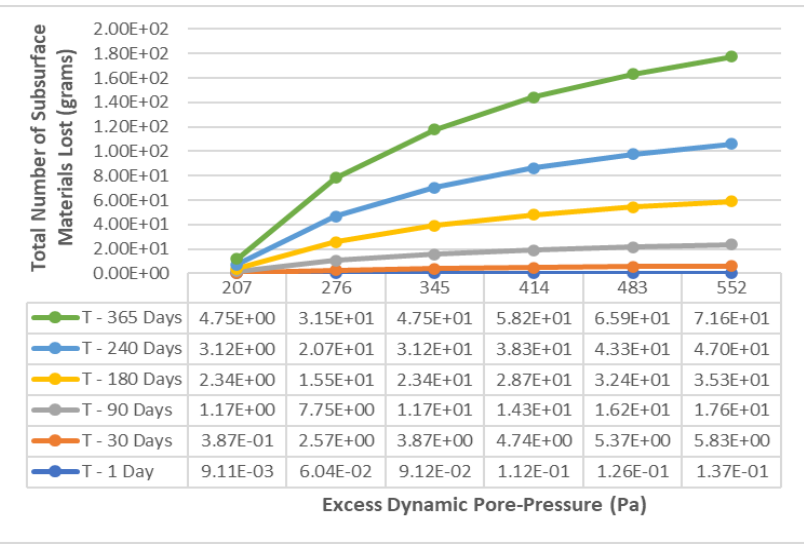

Figure 6. Effect of time on the total number of subsurface materials lost

4.3 Influence of the coefficient of soil erosion on the model's performance

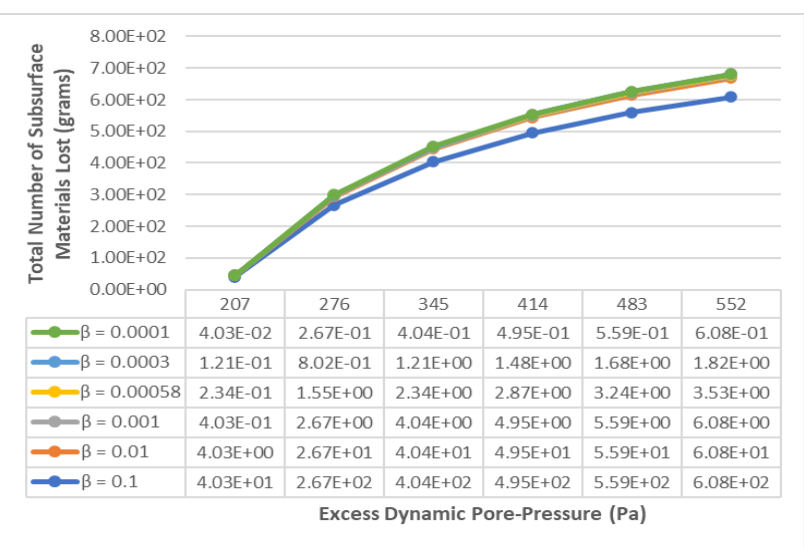

Figure 7. Effect of the coefficient of soil erosion on the total number of subsurface materials lost
We increasingly varied different values for the soil erosion coefficient alongside the excess pore water pressure while keeping other parameters at the same value. We kept the value for the road's height at $0.3 \mathrm{~m}$. The time interval value used was at 180 days. Figure 7 shows the effect of varying the soil erosion coefficient alongside pore water pressure on the total number of materials lost from the roadway's subsurface. The result indicates that an increase in the soil erosion coefficient causes a rise in the total number of materials lost.

\subsection{Influence of road height on the model's performance}

We increasingly varied the road's height alongside increased pore water pressure values while keeping other parameters at the same value. The entered value for the

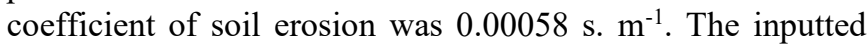
time interval used was at 180 days. Figure 8 shows the effect of varying the road's height alongside pore water pressure on the total number of materials lost from the roadway's subsurface. The result indicates that an increase in the road's height led to a decline in the total number of materials lost from the roadway's subsurface. Subsequently, we still noticed that keeping the road's height at the same value, an increase in the excess pore pressure led to a rise in the total number of materials lost from the roadway's subsurface.

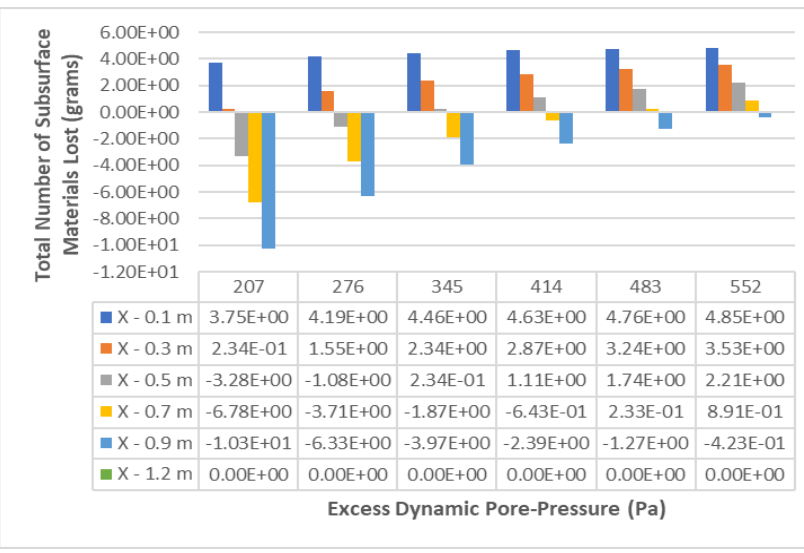

Figure 8. Effect of varying the roadway's height on the total number of subsurface materials lost

\section{CONCLUSIONS}

We reached several conclusions and achieved our aims. We first derived, solved, used past data, and verified a mechanistic mathematical model to show why, how, and when potholes develop on roads from losing its subsurface materials.

Second, our results show why we reached our goals. It is so because we predicted the total number of eroded materials lost or pumped out from a road's subsurface. It is a known precursor to the processes of the displacement of materials, voids, depression, and the formation of potholes on roadways.

Our methods prove that we can use the principles governing how particles migrate or pump out, the technique of separation of variables, and past data to derive, solve, and verify pothole mathematical models.

Next, our literature reviews show why potholes occur on roadways. It shows that potholes arise on roads because of excess traffic-load pressure, poor construction, poor foundation, lack of drains, materials fatigue, use of low quality, and inadequate materials, and the presence of fluid. 
Further, our results show how potholes occur. The application of excess traffic-load pressure increases the porewater pressure. It then triggers the processes of internal erosion, migration of roadway particles upwards, loss of those materials, voids creation, the occurrence of potholes, and then a decline in the roadway's life.

Later, our outcomes prove when potholes occur. It demonstrates that an increase in pore water pressure, time, soil erosion coefficient, and a reduction in the road's height causes more particles to migrate upwards and materials loss. So, when the total number of eroded subsurface materials is high, the road is prone to early potholes development. We can also imply that rising the road's height rises the distance to be moved upwards by the migrating eroded particles.

Finally, this paper provided unique insights into why, when, and how potholes occur on roadways, and it gives engineers, road managers, and modelers better acumen on potholes.

\section{REFERENCES}

[1] Christopher, B.R., Schwartz, C.W., Boudreaux, R., Berg, R.R. (2006). Geotechnical aspects of pavements (No. FHWA-NHI-05-037). United States. Federal Highway Administration.

https://www.fhwa.dot.gov/engineering/geotech/pubs/05 037/05037.pdf.

[2] Jassal, K.S. (1998). Development of potholes from cracks in flexible pavements. Doctoral dissertation, Concordia University.

[3] Ogbezode, J.E., Adeleke, A.I., Adebayo, A.S. (2018). Influence of compressive, tensile and fatigue stresses on asphalt and concrete cement road pavements in Nigeriausing linear elastic theory. Journal of Engineering Research and Reports, 1-19. https://doi.org/10.9734/jerr/2018/v1i39849

[4] Schaefer, V.R., White, D.J., Ceylan, H., Stevens, L.J. (2008). Design guide for improved quality of roadway subgrades and subbases. https://lib.dr.iastate.edu/intrans reports/46.

[5] Kumar, A., Jaiswal, D.K., Kumar, N. (2012). Onedimensional solute dispersion along unsteady flow through a heterogeneous medium, dispersion being proportional to the square of velocity. Hydrological Sciences Journal, 57(6): 1223-1230. https://doi.org/10.1080/02626667.2012.695871

[6] Kumar, A., Jaiswal, D., Kumar, N. (2009). Analytical solutions of one-dimensional advection-diffusion equation with variable coefficients in a finite domain. Journal of Earth System Science, 118(5): 539-549. https://doi.org/10.1007/s12040-009-0049-y

[7] Lewis, K.H., Harr, M.E. (1969). Analysis of concrete slabs on ground subjected to warping and moving loads. Nat Acad Sciences-Nat Research Council-Highway Research Rec, 29: 194-211.

[8] Matei, F., Budiu, V., Dirja, M., Pop, I., Cluj-napoca, M. M. U., Street, M. (2008). Differential equations and their application to the soil moisture study. Bulletin UASVM, Horticulture, 65(2): 594-598.

[9] Mondol, H., Mallick, U., Biswas, M. (2018). Mathematical modeling and predicting the current trends of human population growth in Bangladesh. Modelling, Measurement and Control D, 39(1): 1-7. https://doi.org/10.18280/mmc d.390101
[10] Yadav, R.R., Roy, J. (2019). Solute transport phenomena with input through a plane surface in porous media. Mathematical Modelling of Engineering Problems, 6(4): 557-565. https://doi.org/10.18280/mmep.060411

[11] Chen, X., Zhang, X., Wu, Z. (2019). Analytical solution for one-dimensional transport of particles considering dispersion in deposition kinetics. Geofluids, 2019. https://doi.org/10.1155/2019/1941426

[12] Yesuf, G.Y. (2014). Influence of subsoil conditions on the design and performance of flexible pavements (Issue September). Norwegian University of Science and Technology. http://hdl.handle.net/11250/233254.

[13] Alnedawi, A., Al-Ameri, R., Nepal, K.P. (2019). Neural network-based model for prediction of permanent deformation of unbound granular materials. Journal of Rock Mechanics and Geotechnical Engineering, 11(6): 1231-1242. https://doi.org/10.1016/j.jrmge.2019.03.005

[14] Van Cauwelaert, F., Stet, M., Jasienski, A. (2002). The general solution for a slab subjected to centre and edge loads and resting on a Kerr foundation. International Journal of Pavement Engineering, 3(1): 1-18. https://doi.org/10.1080/10298430290029894

[15] Chang, S. (1965). Infinite beams on an elastic foundation [University of Missouri at Rolla]. https://scholarsmine.mst.edu/masters theses/5204\%0A.

[16] Kuchárová, D., Lajčáková, G. (2017). Moving load effect on concrete slab. Procedia Engineering, 190: 326333. https://doi.org/10.1016/j.proeng.2017.05.345

[17] Omolofe, B.A.B.A.T.O.P.E., Adeloye, T.O. (2017). Behavioral study of finite beam resting on elastic foundation and subjected to travelling distributed masses. Latin American Journal of Solids and Structures, 14(2): 312-334. http://dx.doi.org/10.1590/1679-78253071

[18] Indraratna, B., Singh, M., Nguyen, T.T., Leroueil, S., Abeywickrama, A., Kelly, R., Neville, T. (2020). A laboratory study on fluidization of subgrade under cyclic train loading. Canadian Geotechnical Journal. https://doi.org/10.1139/cgj-2019-0350

[19] Jung, Y.S. (2010). Advancement of Erosion Testing, Modeling, and Design of Concrete Pavement Subbase Layers (Issue August). Texas A\&M University.

[20] Kermani, B. (2018). Experimental and numerical study of subgrade soil migration into pavement subbase and mitigation using geotextile (Issue May) [Pennsylvania State University]. https:/etda.libraries.psu.edu/catalog/15136bzk5102

[21] Agbenyeku, E.E., Muzenda, E., Msibi, M.I. (2017). Diffusion in saturated clayey barrier medium. Procedia Manufacturing, 7: 269-276. https://doi.org/10.1016/j.promfg.2016.12.063

[22] Chihping, K., Chihhao, H., Chinwei, W., Pangliang, L., Derwei, C. (2017). Study on the piping path and mechanism of mud-pumping in railway subgrade. Proceedings of the 19th International Conference on Soil Mechanics and Geotechnical Engineering, Seoul. https://doi.org/10.1007/978-3-319-73568-9 174

[23] Duong, T.V., Cui, Y.J., Tang, A.M., Dupla, J.C., Canou, J., Calon, N., Robinet, A., Chabot, B., De Laure, E. (2014). Physical model for studying the migration of fine particles in the railway substructure. Geotechnical Testing Journal, 37(5): 895-906. https://doi.org/10.1520/GTJ20130145

[24] Fallah, H., Fathi, H.B., Mohammadi, H. (2012). The mathematical model for particle suspension flow through 
porous medium. Geomaterials, 2(3): 57-62. https://doi.org/10.4236/gm.2012.23009

[25] Nguyen, T.T., Indraratna, B. (2019). Mud Pumping under railtracks: Mechanisms, assessments, and Mud Pumping Under Railtracks: Mechanisms, Assessments. Australian Geomechanics Journal, 54(4): 59-80.

[26] Van, W.A. (1985). Rigid Pavement Pumping: (1) Subbase Erosion and (2) Economic Modeling: Informational Report. FHWA/IN/JHRP-85/10. Joint Highway Research Project, Indiana Department of Transportation and Purdue University, West Lafayette. Indiana. https://doi.org/10.5703/1288284314094

[27] Yu, S., Leng, W.M., Teng, J.D., Nie, R.S., Qi, Y. (2016). Analysis of subgrade soil mud pumping model. Electronic Journal of Geotechnical Engineering, 21(24): 7667-7678.

[28] Berlamont, J. (1989). Pumping fluid mud: Theoretical and experimental considerations. Journal of Coastal Research, 5:

195-205. https://www.jstor.org/stable/25735377

[29] Aboubacar, D., Li, W., Tamba, C. (2018). Modeling of internal erosion and initiation of piping phenomenon in a dam. American Journal of Engineering Research, 7(10): 57-64.

[30] Bennacer, L., Ahfir, N.D., Bouanani, A., Alem, A., Wang, H. (2013). Suspended particles transport and deposition in saturated granular porous medium: Particle size effects. Transport in Porous Media, 100(3): 377-392. https://doi.org/10.1007/s11242-013-0220-4

[31] Bonelli, S., Brivois, O., Borghi, R., Benahmed, N. (2006). On the modelling of piping erosion. Comptes Rendus Mecanique, 334(8-9): 555-559. https://doi.org/10.1016/j.crme.2006.07.003

[32] Chetti, A., Benamar, A., Hazzab, A. (2016). Modeling of particle migration in porous media: Application to soil suffusion. Transport in Porous Media, 113(3): 591-606. https://doi.org/10.1007/s11242-016-0714-y

[33] Fell, R., Wan, C.F., Cyganiewicz, J., Foster, M. (2003). Time for development of internal erosion and piping in embankment dams. Journal of Geotechnical and Geoenvironmental Engineering, 129(4): 307-314. https://doi.org/10.1061/(ASCE)10900241(2003)129:4(307) CE

[34] Flayh, M.S., Ahmed, M.D. (2017). The effect of fine particle migration on void ratio of gap graded soil. International Journal of Scientific \& Technology Research, 6(12): 33-41.

[35] Fujisawa, K., Murakami, A., Nishimura, S.I. (2010). Numerical analysis of the erosion and the transport of fine particles within soils leading to the piping phenomenon. Soils and Foundations, 50(4): 471-482. https://doi.org/10.3208/sandf.50.471

[36] Li, Y., Sarishvili, O., Omari, A., Amadi, A., Pu, H. (2017). Colloidal particle deposition in porous media under flow: A numerical approach. European Conference on Design, Modeling and Optimization, Feb 2017, Paris, France, $\quad 7(1)$ : 43-47. https://doi.org/10.7763/IJMO.2017.V7.556

[37] Roy, S., Kumar Bhalla, S. (2017). Role of geotechnical properties of soil on civil engineering structures. Resources and Environment, 7(4): 103-109. https://doi.org/10.5923/j.re.20170704.03

[38] Maria, J., Pietruszka, M. (2009). Derivation of the formula for the filtration coefficient by Application of Poiseuille's law in membrane transport. Acta Societatis Botanicorum Poloniae, 78(2): 93-96. https://doi.org/10.5586/asbp.2009.012

\section{NOMENCLATURE}

$\mathrm{Cp}$ the particle mass concentration in pore fluid, grams

$\mathrm{C}$ the number of materials lost, grams

$\mathrm{C}_{0}$ the initial number of materials lost, grams

$\mathrm{C}_{2}$ the number of roadway subsurface materials lost but trapped in the top part of the stone base and not deposited on the pavement's surface, grams

$\mathrm{C}_{3}$ the total number of roadway subsurface materials lost, grams

$\Delta \mathrm{P}$ excess dynamic pore water pressure across the height of the roadway, $\mathrm{Pa}$

$\mathrm{T}$ time, days

Et erosion rate of the soil material, $\mathrm{kg} . \mathrm{s}^{-1} \cdot \mathrm{m}^{2}$

$\mathrm{V}$ water seepage velocity, $\mathrm{mm} . \mathrm{s}^{-1}$

Vcr critical water seepage velocity, $\mathrm{mm} . \mathrm{s}^{-1}$

M mass of eroded soil, grams

$\mathrm{M}_{0}$ the initial mass of eroded soil from the previous cycle, grams

$\mathrm{N}$ Number of deep pores and cracks

Q flow rate, $\mathrm{m}^{3} . \mathrm{s}^{-1}$

A tire contact area, $\mathrm{m}^{2}$

$\mathrm{R}$ the radius of the flow tube, $\mathrm{m}$

$\mathrm{X}$ height of the roadway's layers above the soil layers, $\mathrm{m}$

\section{Greek symbols}

$\beta \quad$ coefficient of soil erosion, s. $\mathrm{m}^{-1}$

$\tau \quad$ hydraulic shear stress, N. $\mathrm{m}^{-2}$

Tcr critical hydraulic shear stress, N. $\mathrm{m}^{-2}$

$\mu \quad$ dynamic viscosity, N. $\mathrm{s} . \mathrm{m}^{-2}$

$\lambda \quad$ filtration coefficient, $\mathrm{m}^{3} \cdot \mathrm{N}^{-1} \cdot \mathrm{s}^{-1}$ 